\title{
Educação em Saúde e interfaces conceituais: representações de estudantes de um curso de enfermagem
}

\author{
Education in Health and conceptual interfaces: \\ representations of students of a nursing course \\ Educación en Salud y interfacies conceptuales: \\ representaciones de los estudiantes de un curso de enfermería
}

\section{Maristela Inês Osawa Chagas}

Enfermeira. Doutora. Professora da Universidade Estadual Vale do Acaraú (UVA), Sobral, CE.

Lorena Barbosa Ximenes

Enfermeira. Doutora. Professora da Universidade Federal do Ceará, Fortaleza, CE.

Maria Salete Bessa Jorge

Enfermeira. Doutora. Professora da Universidade Estadual do Ceará, Fortaleza, CE.

\section{RESUMO}

Artigo produzido a partir da tese de doutorado: "A Educação em Saúde nos cenários de ensino-aprendizagem: representações de docentes e discentes do curso de enfermagem da Universidade Estadual Vale do Acaraú (UVA)", cujo objetivo foi apreender o sentido das representações elaboradas por professores e alunos do Curso de Graduação em Enfermagem, sobre Educação em Saúde. Os resultados aqui apresentados provêm do Teste de Associação Livre de Palavras. Foi possível inferir que as representações dos estudantes iniciantes estão no núcleo do estabelecido pelo senso comum, considerando a saúde de forma reducionista, própria do modelo biomédico. Os estudantes concludentes evidenciam um conceito positivo de saúde, o que aponta para uma mudança conceitual, influenciada pelas vivências teóricopráticas durante a graduação.

Descritores: Educação em saúde; Programas de graduação em enfermagem; Educação em enfermagem.

\section{ABSTRACT}

Article produced from the a doctoral thesis: "The Education in Health in the scenarios of teach-learning: representations of professors and students from the Nursing School of the University Vale of the Acaraú", whose objective was to apprehend the direction of the representations elaborated for professors and students of the Graduation Nursing Course, about Education in Health. The results presented here come from the Free Test of Words Association. It was possible to infer that the novice students' representations are in the nucleus of what is established as common sense, considering the reductionism concept in health, proper form of the biomedical model. The veterans students evidence a positive concept of health, what points toward a conceptual change, influenced by the theoretician-practical experiences during the graduation.

Descriptors: Education in health; Education, nursing, diploma programs; Education, nursing

\section{RESUMEN}

El artículo ha sido producido de la tesis del doctorado: "La educación en salud en los escenarios de enseñanza-aprendizaje: las representaciones de profesores y estudiantes de un curso de graduación de enfermería de la Universidad Estadual del Vale de Acaraú", cuyo objetivo fue aprehender la dirección de las representaciones elaboradas por los profesores y los estudiantes del curso de graduación en enfermería, sobre la educación en salud. Los resultados presentados vienen de la prueba libre de la asociación de palabras. Fue posible deducir que las representaciones de los estudiantes iniciantes están en el núcleo del establecido para el sentido común, el que considera el concepto reducionista en la salud, lo que es común al modelo biomédico. Los estudiantes veteranos evidencian un concepto positivo de la salud, lo que señala con respecto a un cambio conceptual, influenciado por las experiencias teóricoprácticas durante la graduación.

Descriptores: Educación en salud; Programas de graduación en enfermería; Educación en enfermería.

Chagas MIO, Ximenes LB, Jorge MSB. Educação em Saúde e interfaces conceituais: representações de estudantes de um curso de enfermagem. Rev Bras Enferm 2007 nov-dez; 60(6): 646-50.

\section{INTRODUÇÃO}

É crescente, no discurso contemporâneo do campo da Saúde Coletiva, a referência à idéia de Promoção da Saúde; esta constitui elemento central da chamada nova saúde pública/coletiva, que estende de forma significativa a abrangência das ações de Saúde ao tomar como objeto o ambiente, seja ele local e global, em um sentido amplo(1).

Ainda articulado ao campo da Saúde Coletiva, está o conceito de empowerment comunitário, que se
Submissão: 10/07/2007

Aprovação: 23/10/2007 
refere à participação popular nos assuntos da Saúde e nas ações comunitárias, à aquisição de poder técnico e consciência política para atuar em favor de sua Saúde, bem como o acesso total e contínuo à informação e às oportunidades de aprendizagem nesta área.

Associada à idéia de empowerment comunitário, situamos a Educação que pressupõe o desenvolvimento das pessoas, desde que elas nascem e durante toda a vida nos seus processos de busca de satisfação de suas necessidades. Este desenvolvimento ocorre nas relações sociais de "forma difusa ou organizada, sendo por elas determinado e se traduzem como ação de intervenção política que pode ser utilizada como instrumento de dominação ou libertação".

Sobre a idéia de que a Educação pode ser um instrumento de dominação, Lefevre e Lefevre(2) ${ }^{(2)}$ posicionam contra o termo Educação para a Saúde preferindo Informação para a Saúde, pois partem do princípio de que o termo educar vem do latim educare, que significa conduzir, pressupondo que, por meio da Educação, as pessoas são conduzidas pelo profissional da Saúde (investido de autoridade sanitária) a adotar comportamentos supostamente saudáveis, pois estes profissionais prescrevem comportamentos, uma vez que esta prescrição fortalece e ajuda a reprodução do terreno sanitário como um campo de poder.

Mesmo cientes desta possibilidade, optaremos pela terminologia Educação em Saúde, pois acreditamos e apostamos que é possível vivenciar uma educação como instrumento de libertação(3) que promova o empowerment, ou seja, o fortalecimento das populações para a tomada autônoma de decisões.

Nesse contexto, a Educação em Saúde, assume o estatuto de estratégia de promoção da saúde. Assim, o desafio de que os profissionais de saúde venham a desenvolver a dimensão político-social da educação em saúde exige que, no contexto de ensino-aprendizagem, se efetivem práticas pedagógicas que dêem conta de promover efetivamente a autonomia dos sujeitos.

Assim, interessa-nos conhecer como a Educação em Saúde é percebida pelos discentes do curso de graduação em Enfermagem da Universidade Estado Vale do Acaraú - UVA, de forma que possamos contribuir para a efetivação do SUS e da promoção da Saúde, para a Educação permanente dos professores, para a formação dos estudantes, e, conseqüentemente, para o cuidado e Educação desenvolvidos por eles.

Além do conceito de educação em saúde, acreditamos ser fundamental considerar outros, com os quais a educação em saúde encontra-se articulada. Entendendo a educação em saúde como práxis construída com base em referenciais histórico-culturais, mas também subjetivos, acreditamos que ela pode ser influenciada pelas representações que os sujeitos do processo educativo fazem de si mesmos e de seu "papel", sua "função" no processo.

Assim, além de identificar as representações dos sujeitos do estudo sobre educação em saúde, buscaremos as representações acerca dos conceitos si mesmo e enfermeiro. A exploração deste último conceito deve-se ao fato de que acreditamos que o "papel" e a "função", a que nos referimos anteriormente, está intimamente associada à profissão exercida pelo sujeito.

Entendendo a educação em saúde como instrumento de promoção da saúde, consideramos importante, ainda, conhecer as representações dos sujeitos acerca deste conceito. Acreditamos, ainda, que as representações construídas pelos sujeitos acerca da promoção da saúde são influenciadas pelas noções de saúde e doença nas quais tais construções se apóiam; em função disto, tais noções também serão exploradas neste estudo.

Com arrimo nessas considerações, constitui objetivo do presente estudo: apreender o sentido das Representações elaboradas por alunos do Curso de Graduação em Enfermagem, sobre Educação em Saúde e suas interfaces conceituais.

\section{METODOLOGIA}

A presente investigação, configura-se um estudo de caso, tendo como campo de investigação o Curso de Enfermagem da Universidade Estadual Vale do Acaraú (UVA), situada no Município de Sobral, Ceará, Brasil.

Consideramos como unidades de análise do estudo alunos do referido curso, sendo que somente alunos matriculados no primeiro e último ano do curso foram incluídos. Participaram do estudo 124 sujeitos: 11 professoresenfermeiros (dos 14 inseridos no curso) e 113 (dos 132 alunos matriculados nos quatro semestres escolhidos). Os dados aqui apresentados refere-se, apenas, aos estudantes.

A opção por incluir alunos do primeiro e último anos decorre do fato de que julgamos que a vivência na universidade e nos campos de práticas proporciona experiências com Educação em Saúde que influenciam no conhecimento e atitudes destes alunos.

Para a coleta de dados, realizada no período de novembro de 2005, foi utilizado o Teste de Associação Livre de Palavras (TALP), técnica constituída a partir de estímulos indutores, originalmente desenvolvida por Jung. Esta técnica é utilizada com freqüência em pesquisas em representações sociais à medida que este instrumento permite a atualização de elementos implícitos ou latentes que seriam perdidos ou mascarados nas produções discursivas, já que, potencialmente, traz à tona elementos não "filtrados pela censura".

Neste estudo, elaboramos 07 estímulos indutores, sendo eles: (1) Educação, (2) Saúde, (3) Doença, (4) Educação em Saúde, (5) Enfermeiro, (6) Promoção da Saúde e (7) Si Mesmo. Os dados obtidos pelo TALP foram organizadas pelo software Tri-deux-Mots, método que consiste em representar graficamente a atração entre as variáveis fixas e as variáveis de opiniões, que correspondem às respostas dos sujeitos. Trata-se de uma organização fundamentada na análise fatorial de correspondência (AFC). Todos os dados coletados foram organizados em um banco de dados constituído de variáveis fixas (idade, sexo, ocupação, titulação) e de variáveis de opinião (palavras/ respostas) de todos os sujeitos do estudo com relação a cada estímulo.

Durante o desenvolvimento deste estudou, levamos em consideração os princípios básicos da bioética, preconizados pela Resolução № 196/96 do Conselho Nacional de Saúde. Ressaltemos, ainda, que submetemos a presente pesquisa, como projeto, ao Comitê de Ética em Pesquisa da Universidade Estadual Vale do Acaraú - UVA, para sua apreciação e emissão de parecer, o qual foi aprovado sem restrições de qualquer natureza.

\section{RESULTADOS E DISCUSSÃO}

\section{Representações dos Conceitos Relacionados à Educação em Saúde}

Um contingente de 4.010 palavras foi evocado em resposta aos estímulos indutores do TALP, com a ocorrência de 1736 termos diferentes na coleta de dados pelos 124 sujeitos da pesquisa.

A técnica adotada possibilitou a elaboração de eixos, graficamente representados na figura 1, que evidenciam os resultados apresentados em um campo representacional e distribuídos de maneira oposta sobre os fatores $F_{1}$ e $F_{2}$. Essa formação gráfica reproduz a atração manifesta entre as variáveis fixas (idade, sexo, estudante iniciante, estudante concluinte, professor) e as variáveis de opinião (respostas aos estímulos indutores).

Os fatores podem ser identificados na representação gráfica pela distinção de cores. $O$ fator $F_{1}$, horizontal e de cor vermelha evidencia os resultados mais importantes da pesquisa, em razão do seu valor estatístico, enquanto 0 $F_{2}$, vertical e de cor azul, completa os resultados manifestos no $F_{1}$. Portanto, os fatores $F_{1}$ e $F_{2}$ foram os de maior contribuição, concentrando as principais informações das respostas evocadas pelos sujeitos no TALP. O fator $F_{1}$ abrangeu $53.5 \%$ da variância total de respostas (valor próprio $=0.0429$ ) e 0 segundo fator 21,5\% da variância total de respostas (valor próprio = 0.0173), perfazendo um total de $75 \%$ da variância total dos dados, o que dá confiabilidade às afirmações propostas no capítulo.

Pode-se, objetivamente, visualizar na estrutura do gráfico a oposição entre as representações manifestas pelos discentes concluintes em relação aos estudantes iniciantes. Então, a leitura do gráfico é realizada por meio das 


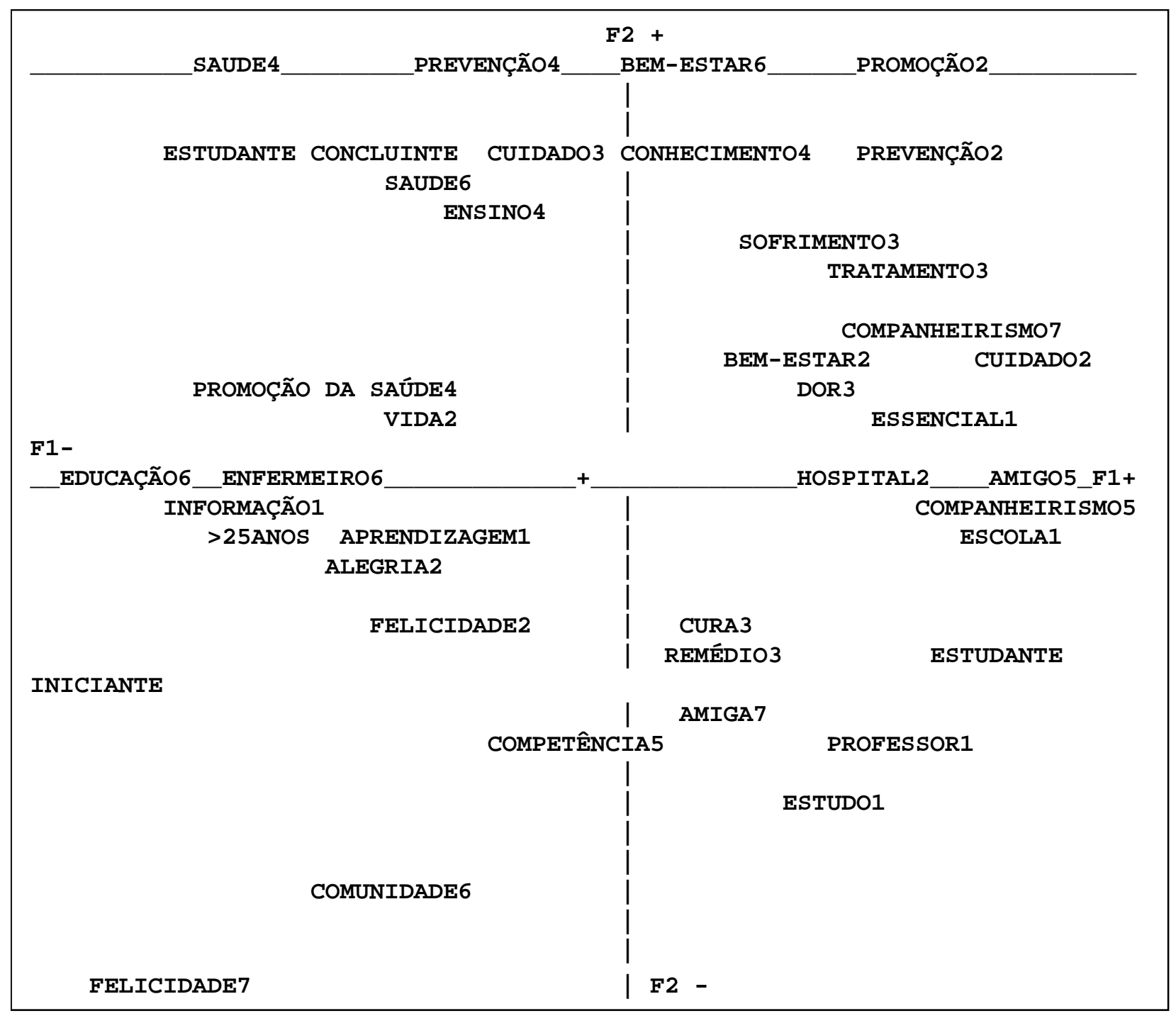

ESTÍMULOS

SUJEITOS

\begin{tabular}{|l|l|l|}
\hline 1. Educação & $\begin{array}{l}\text { 5. Enfermeiro } \\
\text { 2. Saúde }\end{array}$ & Professores e alunos do curso de enfermagem da UVA \\
$\begin{array}{l}\text { 3. Doençáde } \\
\text { 4. Educação em saúde }\end{array}$ & & \\
\hline
\end{tabular}

Figura 1: Análise Fatorial de Correspondência das Representações de discentes do Curso de Enfermagem da UVA sobre conceitos correlacionados com a Educação em Saúde.

palavras evocadas a partir de sua distribuição nos eixos (fatores $F_{1}$ e $F_{2}$ ).

Para fins didáticos, procederemos à análise do fator $F_{1}$ e em seguida do fator $F_{2}$. Convém destacar o fato de que cada resposta vem acompanhada de um número que corresponde ao termo: Contribuição por Fator (CPF), que nesse estudo é tido como significativo para as palavras que obtiveram um CPF acima de 15,6.

Dentre as respostas evocadas, relativas ao estímulo indutor 1 (Educação), destaca-se na fala dos estudantes iniciantes as expressões: essencial $(\mathrm{CPF}=42)$ e escola $(\mathrm{CPF}=26)$. Pode-se observar que tudo o que é representado por esse grupo está no núcleo do estabelecido pelo senso comum, em que Educação é tida como um bem inalienável, intransferível, fundamental para o futuro, adquirindo-se por meio da escola como instituição.

Num extremo oposto estão os sujeitos de mais de 25 anos. O gráfico, todavia, não faz referência à identidade desses sujeitos, fato que nos leva a supor que as evocações estejam distribuídas de forma inespecífica no grupo cuja maioria é constituída por professores e estudantes concluintes. Assim, os sujeitos com idade $>25$ anos (estudantes concluintes) evocaram as palavras: informação (CPF=43) e aprendizagem $(\mathrm{CPF}=25)$ no $\mathrm{F}_{1}$ (eixo 1, lado negativo) para responder ao estímulo 1. É possível, assim, evidenciar que esse grupo associa informação e aprendizagem com aquilo que se espera obter concretamente das relações que tem por finalidade a Educação, seja ela nos espaços formais (escolas e universidades) ou informais (atividades, ações e práticas educativas nos cenários de saúde).

Continuando o trajeto sobre o eixo $\left(F_{1}\right)$, trazemos as evocações manifestas com relação ao estímulo 2 (Saúde). Nesse contexto, destacamos, no lado positivo do $\mathrm{F}_{1}$, o lado dos estudantes iniciantes, as palavras: hospital (CPF=47), cuidado (CPF=41) e bem-estar $(\mathrm{CPF}=16)$. Os achados evidenciam que esses estudantes não conseguem demonstrar o ponto de 
ruptura entre o estado de saúde e de doença, ou seja, para eles, não existe uma fronteira objetiva entre estes dois fenômenos. Isto porque são concepções difíceis de se objetivar, são estados difíceis de mensurar onde termina um e começa 0 outro. Além de pensarem saúde como ausência doença, ainda consideram a saúde de forma reducionista, própria do modelo biomédico, apesar de apontarem o bem-estar como um dos indicadores de saúde, o que provavelmente decorre do conceito de saúde descrito pela OMS como "um estado completo de bem-estar físico, mental e social, e não apenas a ausência de doença ou enfermidade". Em contrapartida, pode-se identificar no extremo oposto (lado negativo do eixo F1), na fala dos sujeitos de mais de 25 anos, um conceito positivo de saúde quando esses relatam alegria (CPF=20) e vida $(C P F=20)$. Estes conceitos estão muito associados a outro - é o de qualidade de vida - que, embora não tenha aparecido no gráfico, foi muito mencionado pelos sujeitos de uma forma geral. A partir desse entendimento, considera-se que a qualidade de vida resulta das relações entre condições objetivas de vida e certas variáveis mais subjetivas e pessoais, cujo resultado é um índice de satisfação e felicidade nos indivíduos. Assim, a qualidade de vida é um conceito que abarca todos os aspectos da vida, tais como experiências dos indivíduos, incluindo saúde, família, trabalho, oportunidades educativas, auto-estima, criatividade, competência, entre outras.

Desta forma, distintas representações da doença foram manifestadas, considerando os diferentes sujeitos em estudo. As representações vão desde o entendimento de saúde associada à doença, inferindo que não se pode pensar saúde de forma desarticulada em relação a doença, até a compreensão de saúde como um dos maiores dos ideais, um bem precioso, ao ser associada a vida e alegria. Assim, é possível identificar nesse campo uma mudança conceitual, além de evidenciar influência por parte do curso de graduação e dos demais cenários de prática, uma vez que a representação mais restrita de saúde partiu dos estudantes iniciantes.

Partindo para as respostas ao estímulo 3 (Doença), o grupo de estudantes iniciantes ressalta dor ( $C P F=18)$ e tratamento $(C P F=18)$, o que nos induz a afirmar que as concepções desse grupo são desenvolvidas e nutridas pela influência do modelo biomédico curativo, aquele que visa essencialmente ao diagnóstico, ao tratamento das doenças e deficiências, que geralmente desencadeiam sofrimento e dor. A corporação médica permeia ainda essa representação da patologia, uma vez que ao médico, principal agente da prática e do conhecimento, cabe o tratamento das enfermidades. Nessa representação aparece uma componente importante para aanálise que se apóia na característica subjetiva da doença, que é a dor, contrapondo-se ao aspecto objetivo, positivista da atenção à saúde, que éo tratamento.

A concepção de doençaé variável, pois os sujeitos com mais de 25 anos não manifestaram uma conceituação homogênea que permitisse aparecer no gráfico. Após analisarmos, porém, o conceito de saúde e doença, percebemos duas perspectivas, uma como visão positiva e outra com a conotação negativa. Consideramos como conceito positivo da saúde aquele que define saúde numa perspectivaampliada, assemelhadaà qualidade de vidaequeéportanto socialmente determinada. Jáa visão negativa de saúde refere-se à concepção limitante, que "enquadra" a saúde nos níveis da ausência de doenças.

Assim, saúde e doença são termos que, ainda hoje, produzem bastante controvérsia, pois algumas pessoas os compreendem como conceitos opostos, isolados, outros os consideram envoltos num continuum e alguns ainda associam o conceito saúde a outros, também complexos, como qualidade de vida e bem-estar.

Ao transpor para a relação saúde/doença, Minayo ${ }^{(4)}$ acentua que é uma noção reveladora da realidade social na qual é construída. É preciso visualizála a partir de substratos econômico, político e cultural no qual vivemos. Numa sociedade capitalista, onde ocorre desigualdade com base na diferenciação de classes, a concepção de saúde/doença, no entanto, está marcada pelas contradições. As representações mais gerais de saúde/doença não são expressões de um desenvolvimento claro das desigualdades nem explicitam os efeitos do modo de produção sobre a vida e a morte dos indivíduos.
Na análise o estímulo 4 (Educação em Saúde), pudemos constatar que os estudantes iniciantes não evocaram nenhum conceito relacionado com a Educação em Saúde de maneira homogênea e significante o suficiente para ser visualizado no gráfico, visto que não vivenciaram experiências teóricopráticas que permitissem a internalização desta dimensão conceitual. Já o grupo opositor a este no gráfico, que apresentou RS homogêneas, ou seja, os sujeitos de mais de 25 anos (alguns estudantes concluintes), relaciona a Educação em Saúde com Promoção da Saúde (CPF=33) e com Saúde (CPF=30). O grupo se refere a Educação em Saúde como uma das estratégias promotoras de saúde, daí a forte evocação da extensão promoção da saúde e, por conseguinte, saúde.

Seguindo, ainda, a interpretação do gráfico em seu eixo principal $\left(F_{1}\right)$, tomamos agora o estímulo 5 (Enfermeiro) como ponto para discussão. Observa-se nas evocações produzidas ante o estímulo indutor a presença das palavras companheirismo ( $C P F=90)$ e amigo ( $C P F=59)$ evocadas pelos estudantes iniciantes. Nesse contexto, as respostas podem ser traduzidas como conceitos que nos remetem à dimensão psicológica e afetiva. $O$ outro grupo, entretanto sequer se refere a "enfermeiro", embora seja constituído, predominantemente, por estudantes concluintes e, neste suposto, se esperaria uma concepção mais ampliada de enfermeiro.

Aspecto relevante pode ser apontado no estímulo 6 (Promoção da Saúde). Os sujeitos maiores de 25 anos associam esse tema com Educação (CPF=97), aí incluídos a Educação em Saúde e Enfermeiro (CPF=23). Percebemos que, para este grupo, não há diferença significativa entre Promoção da Saúde e Educação em Saúde, pois, quando se pensa em um, automaticamente vem à mente o outro e vice-versa. Já os alunos iniciantes ficaram "silenciosos" no que concerne "Promoção da Saúde", assim a "Educação em Saúde", de forma que não configuraram no gráfico seus posicionamentos, em ambos os casos. Chamamos a atenção para o fato de que os estudantes iniciantes ficaram emudecidos, num ato de coerência pois, como responder a um estímulo do qual pouco ou nada se ouviu falar?

Em relação ao estímulo 7 (Si Mesmo), os estudantes iniciantes se descrevem como pessoas companheiras. Nesse sentido, o termo companheirismo ( $\mathrm{CPF}=27$ ) indica uma qualidade pessoal importante para a elaboração da auto-imagem mediante a capacidade de realizar atividades durante o processo de cuidado, bem como na vida cotidiana.

Em contraste à representação do grupo anterior, os maiores de 25 anos vêem-se como pessoas felizes e, assim, o termo felicidade (CPF=55) reflete predicativo subjetivo sobre si mesmos, atribuindo forte componente de satisfação profissional e pessoal à sua representação. Apesar de ser uma alusão positiva ao estímulo, a evocação aparece muito distante dos eixos principais, reduzindo, assim, seu valor representacional.

Adicionalmente, traremos à discussão os resultados apresentados no eixo $\left(F_{2}\right)$ que, apesar de menos relevantes em termos estatísticos, contribui de maneira complementar para a apreensão das Representações da Educação em Saúde pelos sujeitos em estudo.

A estrutura assumida no gráfico pelo grupo dos estudantes concluintes revela que não foram evidenciadas respostas para o estímulo 1 , visto que as evocações foram distribuídas de forma inespecífica no grupo, não aparecendo palavras que expressam uma representação de educação para esse grupo.

Em resposta ao estímulo 2 (Saúde), o grupo dos estudantes concluintes diz, prioritariamente, promoção ( $C P F=42$ ) e prevenção ( $C P F=27)$, o que nos induz à interpretação de que há no imaginário desse grupo uma conduta de ações que eles aprenderam que devem ser executadas para se obter a saúde, muito discutidas na sua formação, que se trata das ações de promoção da saúde e de prevenção de doenças. Evidencia-se, ainda, que uma palavra freqüentemente evocada como representativo de saúde foi felicidade $(\mathrm{CPF}=19)$. Assim, a idéia de saúde encontra-se muito próxima do que cada um considera "sentir-se bem". Dessa forma, percebemos que ainda temos definido saúde como oposto de doença, como algo que somente percebemos quando sentimos a sua ausência. Assim, quando pensamos nas práticas e nas representações que os indivíduos, grupos e coletividades apresentam a 
respeito de saúde e doença, notamos que existe uma multiplicidade de justificativas, explicações e discursos relacionados com a idéia de que a expressão saúde se relaciona ao fato de não precisar tomar medicamentos nem utilizar os serviços de saúde. Caso contrário, a resposta será de manifestação de doença.

No que diz respeito ao estímulo 3 (Doença), o grupo dos estudantes concluintes menciona os vocábulos sofrimento $(\mathrm{CPF}=19)$ e cuidado $(\mathrm{CPF}=64)$, numa idéia de que doença significa sofrimento e quem sofre necessita, conseqüentemente, de cuidados.

No que concerne ao estímulo 4 (Educação em Saúde), há uma afirmação, por parte dos estudantes concluintes, das conseqüências positivas que esta ação pode trazer tais como: conhecimento $(\mathrm{CPF}=24)$, prevenção $(\mathrm{CPF}=135)$ e saúde (CPF=62); bem como apontam a via principal para se chegar até essas conseqüências: 0 ensino (CPF=17). Estes resultados enfatizam o já dito acerca das concepções dos alunos concluintes; ou seja, não se evidenciam concepções próativas na perspectiva de um ensino que considere a educação em saúde enquanto estratégia transformadora para a promoção da saúde.

Com relação ao estímulo Enfermeiro, de número 5, o grupo de alunos concluintes não conseguiu evidenciar uma representação para que configurasse na figura.

Aspecto relevante que pode ser apontado no estímulo 6, "Promoção da Saúde", diz respeito ao fato de que os estudantes concluintes relacionam este conceito com saúde (CPF=35) e bem-estar ( $C P F=58$ ) levando-nos a crer que associam o estímulo ao novo paradigma da saúde, fundamentado na qualidade de vida. Já os professores, associaram este estímulo à comunidade (CPF=56), reforçando uma representação de que promoção da saúde está associada de forma restrita a atenção básica e, por conseguinte, a comunidade, uma vez que esta temática (promoção da saúde) é mais discutida e divulgada nas pesquisas da saúde pública/coletiva. Assim, a representação é de uma visão reducionista da dimensão, porém é fato que, nesse âmbito da atenção à saúde discute-se esse assunto de maneira mais consistente. Daí a forma como o assunto passa a ser comunicado.

Em relação ao sétimo e último estímulo (si mesmo), os estudantes iniciantes não têm uma representação elaborada sobre si mesmos, talvez porque ainda estão em decurso de autoconhecimento e prestes a se inserir no mercado de trabalho.

\section{CONSIDERAÇÕES FINAIS}

Distintas representações da doença foram manifestadas, considerando os diferentes sujeitos em estudo. As representações dos estudantes iniciantes está no núcleo do estabelecido pelo senso comum, em função da ausência de vivências teórico-práticas no campo da saúde.

No que se refere à saúde, os estudantes iniciantes a consideram de forma reducionista, própria do modelo biomédico. Em contrapartida, os sujeitos com mais de 25 anos evidenciam um conceito positivo de saúde. Assim, é possível identificar nesse campo uma mudança conceitual, além de evidenciar influência por parte do curso de graduação e dos demais cenários de prática. Percebe-se, entretanto, que não há a representação de saúde como um direito dos cidadãos e da coletividade, e, uma vez que as representações constituem modos de pensar, fazer e sentir, faz-se necessário ampliar as representações sobre saúde no cenário em estudo.

\section{REFERÊNCIAS}

1. Czeresnia DE, Freitas CM. Apresentação. In: Czeresnia DE Freitas CM. Promoção da saúde: conceitos, reflexões, tendências. Rio de Janeiro (RJ): Fiocruz; 2003.

2. Lefevre $F$, Lefevre AMC. Promoção de saúde: a negação da negação. Rio de Janeiro (RJ): Vieira \& Lent; 2004.
3. Araújo IMB. Aprendem doença, educam para a saúde: Influência da formação, em futuros profissionais de saúde, em concepções saúde/doença, educação para a saúde e sua implementação (dissertação). Braga (POR): Universidade do Minho; 2004.

4. Minayo MCS. Interdisciplinaridade: funcionalidade ou utopia? Rev Saúde Sociedade 1994; 2(1): 42-64. 